\title{
Amorphous Silica and Carbon-rich nanotemplated surfaces as model interstellar dust surfaces for laboratory astrochemistry
}

\author{
Natalia Pascual ${ }^{1,2}$, A. Dawes ${ }^{1}$, F. Gonz'alez-Posada ${ }^{3}$, D. Chakarov ${ }^{2}$, \\ N. Thompson ${ }^{4}$, N. J. Mason ${ }^{1}$ and H. J. Fraser ${ }^{1}$ \\ ${ }^{1}$ Department of Physical Sciences, The Open University, Milton Keynes, United Kingdom, \\ email: natalia.pascual@open.ac.uk \\ ${ }^{2}$ Dept. Applied Physics Chalmers University of Technology, Gothenburg, Sweden, ${ }^{3}$ Université \\ de Montpellier, France, ${ }^{4}$ Universtiy of Leeds, United Kingdom
}

\begin{abstract}
Traditional laboratory studies on dust-ice systems have proved how the nature of the dust surface significantly affects ice structure and reactivity. Although the surface composition effects have been widely studied recently, no attention has been paid to the dust sizes. We show how dust the grains size and topography, as well as their composition, affects their interaction with light and the morphology of water ice mantles on top of them.
\end{abstract}

Keywords. ISM dust, astrochemistry

Dust grains play a major role in the physical and chemical conditions of the interstellar medium (ISM). They affect its temperature balance and act as a catalytic surface for its chemical reactions (Tielens 2013). Experimental studies on surface astrochemistry have provided a great understanding of chemical evolution in the ISM. What traditional studies are still lacking is the ability to account for effects arising from the discrete nano-scale of ISM grains, which might include changes to electronic structure, optical properties and surface-kinetics in comparison to bulk materials. The question arises: to what extent are the chemical and optical properties of interstellar ices affected by the size, morphology and material of the underlying ISM dust?

We have designed, fabricated (by means of colloiday lithography, Hulteen et al. 1995) and characterised a set of nano-structured surfaces, where nanoparticles, representative of ISM grains, are adhered to an underlying support substrate. The optical characterisation of these surfaces shows how their interaction with light is modified by several factors such as their composition and their topographic features in light scattering and absorption, with direct implications in astronomy. In our spectroscopic study of icy mantles deposited on these analogues, we showed how both, the ice uptake and the ice morphology change for thin ice layers on top of different substrates, evidencing how the substrates have to be characterised not only in terms of composition but also in size and roughness.

\section{References}

Tielens, A. G. G. M. (2013). The molecular universe. Reviews of Modern Physics, 85, 1021-1081. Hulteen, J. \& Vanduyne, R. (1995). Nanosphere Lithography - A Materials General Fabrication Process For Periodic Particle Array Surfaces. Journal of vacuum science 83 technology AVacuum surfaces and films, 13(3, 2), 1553-1558. 